\title{
Zusammenarbeit mit anderen Fachärzten
}

\author{
Ein Gynäkologe und eine Radiologin mussten sich vor dem Landgericht Koblenz verantworten. Ihnen wurde \\ vorgeworfen, eine Brustkrebserkrankung nicht rechtzeitig erkannt zu haben.
}

$\mathrm{D}$ ie 50 -jährige Patientin ertastete im Mai 2006 einen Knoten in der rechten Brust. Der Gynäkologe stellte einen „abklärungsbedürftigen unklaren Verdichtungsbezirk ohne Umgebungsreaktion und Axilla-Auffälligkeit“ fest und überwies die Patientin zur Mammografie und Mammasonografie an die Radiologin. Diese stellte „unklare Strukturen "fest, klassifizierte den Befund nach BI-RADS III und empfahl eine Kontrollmammografie in sechs Monaten. Die Kontrolle ergab neuerlich einen unklaren Befund, mit Ausnahme einer Mamilleneinziehung war eine wesentliche Veränderung nicht feststellbar. Eine nunmehr empfohlene kernspintomografische Untersuchung ergab ein aggressives, schnell wachsendes Mammakarzinom.

Die Patientin warf der Radiologin eine fehlerhafte Klassifizierung der Befunde und daraus resultierend eine fehlerhafte Empfehlung zur Kontrolle vor. Zudem hätte sich der Gynäkologe wegen der unklaren Diagnose nicht auf die radiologische Empfehlung verlassen dürfen, sondern weitere diagnostische Maßnahmen, etwa eine Biopsie, einleiten müssen. Der Klageschrift legte die Klägerin ein ihre Vorwürfe bestätigendes Gutachten des MDK bei.

\section{So sah das Gericht den Fall}

Das Landgericht Koblenz hat - sachverständig beraten - die Klage abgewiesen (Urteil vom 11.05.2011, Az. 10 O 231/09), bestätigt durch das Oberlandesgericht Koblenz, Beschluss vom 23.12.2011, Az. 5 U 688/11). Den Ärzten könnten keine Sorgfaltspflichtverstöße vorgeworfen werden. Aufgrund der erhobenen Befunde sei die getroffene Klassifizierung in BI-RADS III nachvollziehbar und die daraus leitliniengerecht ermittelte Empfehlung zur Kontrolle in sechs Monaten nicht zu beanstanden. Die abweichende Auffassung im Gutachten des MDK überzeuge nicht, zumal es an einer hinreichenden Begründung mangele und allein auf einer wiederum nicht begründeten Rückbetrachtung beruhe. Es sei auch nicht erkennbar, weshalb der Gynäkologe seinerseits ein abweichendes Vorgehen hätte veranlassen sollen. Vielmehr habe sich dieser auf die Diagnose der Radiologin verlassen dürfen. Auch insoweit seien die Ergebnisse des Gutachtens des MDK nicht nachvollziehbar, zumal dort weder dargelegt noch begründet werde, gegen welche Pflichten der Gynäkologe verstoßen haben solle.

\section{Was bedeutet das Urteil für den klinischen Alltag?}

Bei arbeitsteiligem Handeln von Ärzten unterschiedlicher Fachrichtungen gilt der Vertrauensgrundsatz. Hiernach darf ein Arzt grundsätzlich auf die fachliche Richtigkeit des Handelns und der erhobenen Befunde des Kollegen vertrauen. Es bestehen keine wechselseitigen Überwachungs- oder Kontrollpflichten. Drängen sich hingegen Zweifel auf, ist diesen nachzugehen (vgl. gynäkologie + geburtshilfe 2/2008, S. 64).

Der Umstand, dass ein Gutachten des MDK in einem gerichtlichen Verfahren nicht bestätigt wird, stellt kein vereinzeltes Phänomen dar. Das Landgericht Gießen (Urteil vom 17.04.2009, Az. 3 O 558/07) führte insoweit bereits mit erfreulicher Klarheit aus: „Dass der Privatgutachter [Name] eine andere Auffassung vertritt, steht den Feststellungen

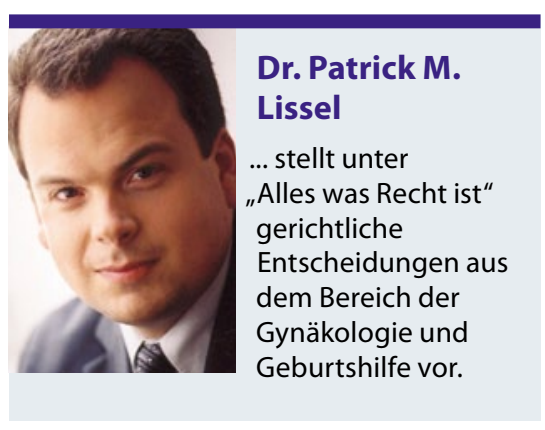

des gerichtlichen Sachverständigen nicht entgegen. Der gerichtliche Sachverständige hat sich intensiv mit den Ergebnissen des Privatgutachtens auseinandergesetzt und mit nachvollziehbarer Begründung ausgeführt, warum die Einschätzung des [Name] aus fachlicher Sicht nicht haltbar ist. Im Übrigen häufen sich bei der Kammer in der letzten Zeit Fälle, in denen Patienten durch fachlich zweifelhafte und von einer starken Belastungstendenz geprägte Gutachten, die durch den MDK veranlasst werden, in die irrige Annahme versetzt werden, eine Arzthaftungsklage habe Aussicht auf Erfolg. Infolgedessen ist es an der Tagesordnung, dass seitens der Gerichte bestellte Sachverständige den Einschätzungen der MDK-Gutachter widersprechen." Dem ist nichts hinzuzufügen.

\section{Dr. Patrick M. Lissel, LL.M.}

Rechtsanwalt und Fachanwalt für

Medizinrecht

Ratzel Rechtsanwälte

Ottostraße 1

80333 München 